\title{
Effects of pulsed electromagnetic fields on histomorphometry and osteocalcin in disuse osteoporosis rats
}

\author{
Baolin $\mathrm{Li}^{\mathrm{a}, 1}$, Jiaqi Bi ${ }^{\mathrm{a}, 1}$, Wei Li ${ }^{\mathrm{a}, 1}$, Sainan Huang ${ }^{\mathrm{a}, 1}$, Shuangli Zhang ${ }^{\mathrm{a}}$, Jian'gang Zhao ${ }^{\mathrm{b}}$, \\ Qinggang Menga,* and Jianfeng Fei ${ }^{\mathrm{b}, *}$ \\ a Department of Orthopaedic Surgery, Harbin Medical University, The First Hospital of Harbin City, \\ Harbin, Heilongjiang, China \\ ${ }^{\mathrm{b}}$ Department of Surgery, The First Hospital of Harbin City, Harbin, Heilongjiang, China
}

\begin{abstract}
.
OBJECTIVE: Bone histomorphometry and the concentration of the serum osteocalcin expression were observed in disuse osteoporosis (DOP) rats in order to explore the mechanism of pulsed electromagnetic fields in treating DOP.

METHODS: Female SD rats, weighing $250 \sim 280 \mathrm{~g}$, were randomly divided into 4 groups: a control group and three experimental groups. The right hindlimbs of all the rats were immobilized by tibia-tail fixation, except for those in the INT group. The $A L N$ group rats were given an alendronate sodium $\left(1 \mathrm{mg} \mathrm{kg}^{-1} \mathrm{~d}^{-1}\right)$ treatment, and the rats in the PEMF group received PEMF irradiation. Bone histomorphometry and the concentration of serum osteocalcin expression were detected in $2,4,8$, and 12 weeks.

RESULTS: Four weeks after modeling, as compared with the DOP group, the \% Tb-Ar and Tb-N in the ALN group were increased, and the difference was significant $(P<0.05$ or $P<0.01)$. At 8 weeks, as compared with the DOP group, the $\% \mathrm{~Tb}-\mathrm{Ar}$ and Tb-N in the ALN group and the PEMF group both increased, and there was a significant difference $(P<0.05$ or $P<$ 0.01). At 12 weeks, as compared with the DOP group, the BGP serum concentration of the ALN group was reduced, and there was a statistical difference $(P<0.05)$.

CONCLUSION: Pulse electromagnetic field with drug can prevent disuse osteoporosis by changing the bone microstructure. In the long run, PEMF improves the mechanical performance of biological structures better than alendronate sodium does. PEMF may influence the process of bone remodeling by promoting the level of osteocalcin.
\end{abstract}

Keywords: Pulse electro magnetic fields, disuse osteoporosis, donatello sodium, metrology static parameters

\section{Introduction}

Osteoporosis is a systemic skeletal disease characterized by low bone mass and micro-architectural deterioration, which increases the risk of fracture. Disuse osteoporosis (DOP) is a kind of secondary osteoporosis that can be caused by various reasons, such as motion ability restriction or dysfunction or

\footnotetext{
${ }^{1}$ These authors contributed equally to this work.

${ }^{*}$ Corresponding authors: Jianfeng Fei, Department of Surgery, The First Hospital of Harbin City, Harbin, Heilongjiang, China. Tel.: +86 45184884409; Fax: +86 45184884409; E-mail: hsn1012@163.com; Qinggang Meng, Department of Orthopaedic Surgery, Harbin Medical University, The First Hospital of Harbin City, 7 Harbin, Heilongjiang, China E-mail: doctorbaolin@163.com.
}

0928-7329/17/\$35.00 (c) 2017 - IOS Press and the authors. All rights reserved

This article is published online with Open Access and distributed under the terms of the Creative Commons Attribution NonCommercial License (CC-BY-NC 4.0). 
motion system damage. DOP is frequently seen in hemiplegia, fracture, long-term bed rest, and space flight [1,2]. In recent years, with the increase in the number older individuals and the use of mechanized transportation, the high incidence of disuse osteoporosis is becoming a significant problem in clinical medicine.

DOP can result in severe damage to the bone microstructure in addition to lost bone mass. The threedimensional structure of the trabecular bone and the degree of connection are known as bone microstructure. At present, the bone histomorphometry method has been refined for conducting a quantitative analysis of bone microstructure in the study of DOP.

There is a long history of using the effects of pulsed electromagnetic fields (PEMFs) to treat osteoporosis for a long history. Since the discovery of strain-generated potentials in bone and their potential influence on bone formation and remodeling, the effects of direct and indirect electrical and magnetic stimulation on bone metabolism in bones have been studied in numerous experiments. Although the effect of PEMFs on bone formation and remodeling has been investigated in several studies, no consistent results have been obtained [3-5].

To clarify these conflicting results, a tibial-tail fixation model in rats was established. The model induces an osteopenia in rats, which primarily reduced bone formation rather than increased bone resorption [6,7]. Furthermore, it was proven that the model had reduced osteoblast populations and bone formation in the affected rat hind limb long bones [8]. The author investigated the effect of 60 days of PEMFs exposure on disuse osteoporosis rats. Bone morphology metrology and BGP concentration in the serum were also evaluated in order to discuss the possible mechanism of PEMF curing disuse osteoporosis.

\section{Materials and methods}

\subsection{Animals}

$\mathrm{SD}$ rats of clean grade, female, and weight of $250 \mathrm{~g} \sim 280 \mathrm{~g}$ were provided by the experimental animal center of Jilin University's Bethune School of Medicine, Certificate No. SCXK-Ji 2007-0003. The animals were kept at a specific pathogen free (SPF) animal house at a controlled temperature (22 $\pm 2^{\circ} \mathrm{C}$ ), under $12 \mathrm{~h} \mathrm{light/dark} \mathrm{cycles.} \mathrm{The} \mathrm{experimental} \mathrm{procedures} \mathrm{were} \mathrm{approved} \mathrm{by} \mathrm{the} \mathrm{Committee} \mathrm{of}$ Animal Administration of Harbin Medical University.

\subsection{PEMF system}

The PEMF exposure system developed for this study was composed of a waveform generator and Helmholtz coil. The waveform generator generated $10 \mathrm{~Hz}$ repetitive single pulse waveforms. The Helmholtz coil used for the treatment exposures consisted of a $240 \mathrm{~mm}$ diameter, a $0.9 \mathrm{~mm}$ diameter copper wire, and 200 turns. The current passing through the wires was $1 \mathrm{~A}$ with a frequency of $10 \mathrm{~Hz}$. The Helmholtz coils produced a magnetic field with a magnetic field intensity of 15 GS in the center between the coils where the animals received the exposure. Figure 1 illustrates the PEMF circuit diagram used in this study. 


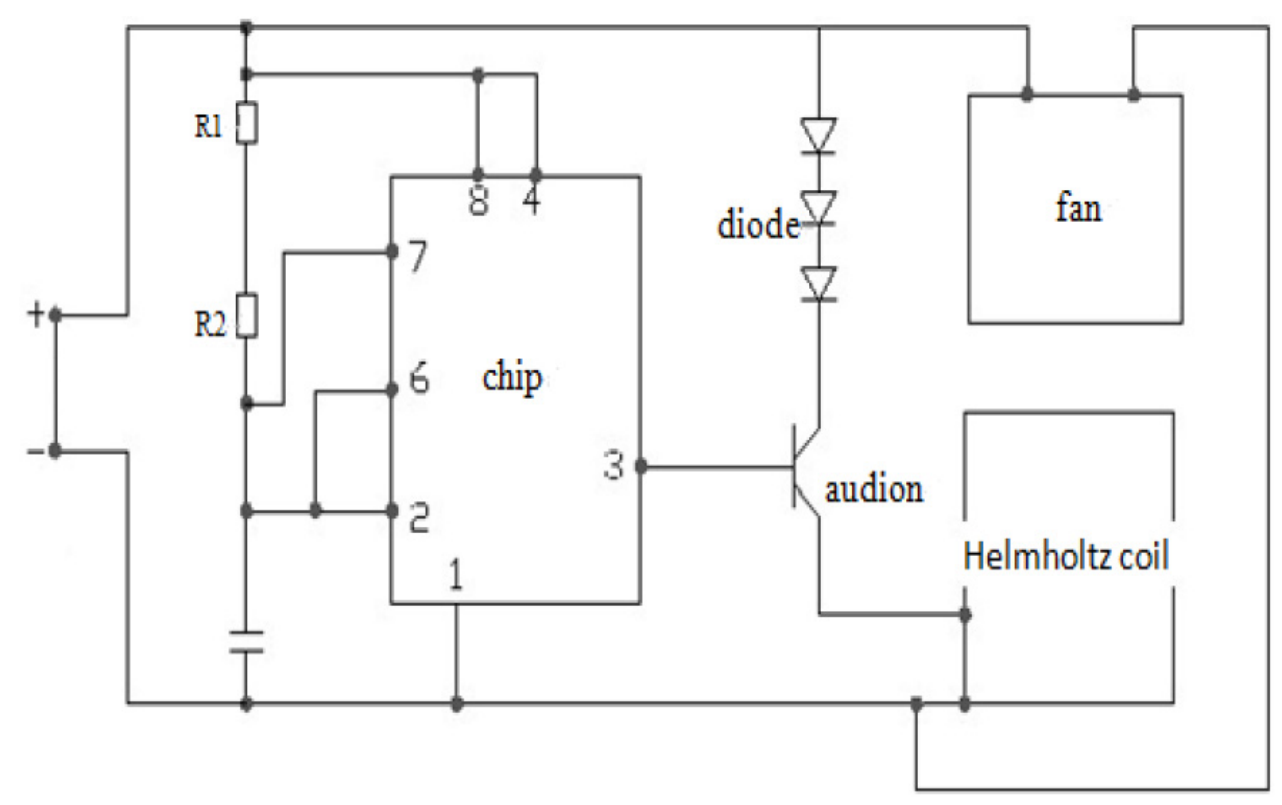

Fig. 1. The PEMF circuit diagram used in the study.

\subsection{PEMF treatment protocols and model preparations}

The rats were divided into four groups according to body mass random sampling, with 20 rats in each group: the intact (INT) group, DOP group, Alendronate sodium (ALN) group, and PEMF group. The disuse osteoporosis rat models were established by modified tibial-tail fixation braking, except for the INT group. The DOP model establishment method was improved on the basis of traditional tibialtail fixation [4]. The rats were anesthetized by an intraperitoneal injection of $30 \mathrm{mg} \mathrm{kg}^{-1}$ pentobarbital sodium. Medical tape was wound on the tail at a distance of about $1 \mathrm{~cm}$ from the anus, and an appropriate diameter medical wire was passed through the right hind tibia distal around $1 \mathrm{~cm}$ from the anus. The hind limbs were fixed to the tape wrapping position. It is advisable to securely fix the legs so that they cannot easily slip. Medical adhesive tape can effectively reduce oppression as well as increase the fixed stress surface. The tail medical steel wire tension was regulated with the rats' growth. All animals received a standard rat diet (calcium and phosphorus contents were $0.51 \%$ and $0.42 \%$, respectively) and were permitted free access to water. The INT group rats were fed with DOP average rat feed consumption in order to avoid weight differences influencing the results of the evaluation.

In order to determine a successful model of DOP, 5 rats were randomly selected for dual energy X-ray absorptiometry (small animal software) of the right proximal femur bone density scans two weeks after the right lower limbs were broken (data not shown).

The ALN group rats were given alendronate sodium $\left(1 \mathrm{mg} \mathrm{kg}^{-1} \mathrm{D}^{-1}\right.$, Alendronate, Hangzhou MSD Pharmaceutical Co., Ltd.). PEMF group rats were given PEMF 40 min D-1 irradiation treatment, with magnetic field parameters of $3.82 \mathrm{mT}$ and $10 \mathrm{~Hz}$.

\subsection{Bone histomorphometry and serum BGP concentration determination}

At 2, 4, 8, and 12 weeks after treatment, 5 rats in each group were sacrificed to detect both bone histomorphometry and serum BGP concentration. Blood samples were taken from the rats' right ventricle. The right tibia was fixed with $4 \%$ paraformaldehyde. The serum was collected after the blood was 
centrifuged for $3000 \mathrm{r} / \mathrm{min}$. The serum was then stored in $-70^{\circ}$ low temperature refrigerators until determination. The BGP concentration was detected with the ELISA method (RAT OT/BGP ELISA assay kit, Wuhan Boster Company). The tibia were sliced and then observed using a micro electron microscope (AMRAY1000B scanning electron microscope, America). The bone histomorphometry was measured with a Q550CW image processing and analysis system (Leica, Germany). The bone histomorphometry parameters, including bone total surface area (T-Ar), trabecular bone area (Tb-Ar), and trabecular bone circumference (Th-Pm), were directly detected. The trabecular bone area percentage (\% Tb-Ar), trabecular thickness $(\mathrm{Tb}-\mathrm{Th})$, trabecular number $(\mathrm{Tb}-\mathrm{N})$, and trabecular separation ( $\mathrm{Tb}-\mathrm{Sp})$ can be separately calculated according to the following formulas:

$$
\begin{aligned}
\% \mathrm{Th}-\mathrm{Ar}= & \mathrm{Tb}-\mathrm{Ar} / \mathrm{T}-\mathrm{Ar} \times 100 ; \mathrm{Tb}-\mathrm{Th}=(2000 / 1.199) \times \mathrm{b}(\mathrm{Th}-\mathrm{Ar} / \mathrm{Tb}-\mathrm{Pm}) ; \mathrm{Tb}-\mathrm{N}=(1.199 / 2) \\
& \times(\mathrm{Tb}-\mathrm{Pm} / \mathrm{T}-\mathrm{Ar}) ; \mathrm{Tb}-\mathrm{Sp}=(2000 / 1.199) \times(\mathrm{T}-\mathrm{Ar}-\mathrm{Tb}-\mathrm{Ar}) / \mathrm{Tb}-\mathrm{Pm}
\end{aligned}
$$

\section{Statistical analysis}

The statistical analyses were performed with SPSS statistics 15.0. The data were analyzed using oneway ANOVA with Bonferroni post hoc tests for multiple comparisons. A value of $p<0.05$ was considered statistically significant. The results are expressed as mean values with standard deviations.

\section{Result}

\subsection{Bone morphology metrology static parameters of observations}

Two weeks after treatment, as compared with the DOP group, the \% Tb-Ar and Tb-N of the ALN group were significantly increased, whereas the $\mathrm{Tb}-\mathrm{Sp}$ was reduced, and the result was significantly different $(P<0.01)$. As compared with the DOP group, the parameters of the PEMF group were not significantly different. Four weeks after curing, as compared with the DOP group, the \% Tb-Ar and Tb$\mathrm{N}$ of the ALN group were significantly increased, whereas the Tb-Sp was reduced, and the result was significantly different $(P<0.01)$. Additionally, Tb-Th was only slightly different. As compared with the DOP group, the \% Tb-Ar and Tb-N of the REMF group increased, and the result was significantly different $(P<0.05$ or $P<0.01)$. The Tb-Th and Tb-Sp were only slightly different. Eight weeks after curing, as compared with the DOP group, the \% Tb-Ar and Tb-N of the ALN group and PEMF group both increased, whereas the Tb-Sp was reduced, and the result was significantly different $(P<0.05$ or $P<0.01)$. At 12 weeks, as compared with the DOP group, the Tb-Ar and Tb-N of the ALN group and the PEMF group both increased $(P<0.01)$, whereas the Tb-Sp was reduced, and the result was significantly different $(P<0.01)$. The Tb-Th was a little higher but the difference was not significant. Figures 2 and 3 give the results.

\subsection{Determination of serum BGP concentration}

After modeling, as compared with the INT group, the rest of the groups were significantly increased. Two weeks after modeling, as compared with the DOP group, the ALN group and the PEMF group did not exhibit any obvious differences. Four weeks after modeling, as compared with the ALN group, the PEMF group's BGP serum concentration was obviously increased $(P<0.01)$. Eight weeks after 

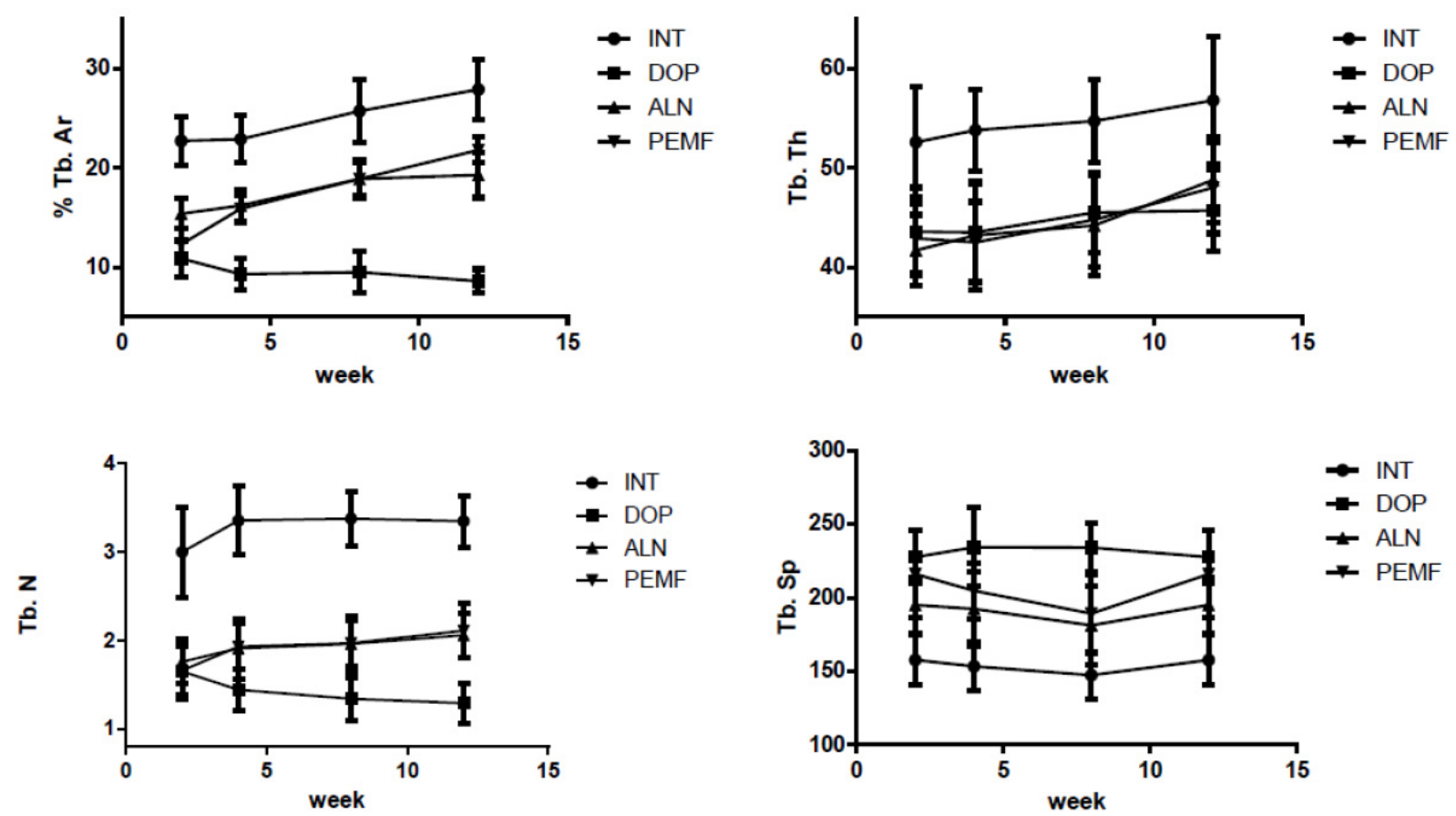

Fig. 2. The variation of the bone histomorphometry during the PEMF treatment.

modeling, the serum BGP concentration of the PEMF group continued to rise. As compared to the DOP group, the ALN group's BGP serum concentration reduced, which was a significant difference $(P<$ 0.05). As compared with the DOP group and ALN group, the PEMF group's serum BGP concentration obviously increased, which was a significant difference $(P<0.01)$ (Fig. 4).

\section{Discussion}

DOP is a type of secondary osteoporosis. It directly results in lower bone mineral content due to body long-term braking, disuse, restricted movement of the body, or its inability to bear load. It often occurs in long-term bedridden or disabled patients and in astronauts in weightlessness [9-12]. To address DOP, the major treatment strategies focus on improving osteoblast activity and reducing bone resorption. Relatedly, many studies reported that PEMF could stimulate the proliferation and differentiation of osteoblasts $[13,14]$; however, the effect of different magnetic field conditions on cell proliferation and differentiation resulted in radically different results. This experiment, using the conditions in reference $[15,16]$ and homemade equipment, used $3.82 \mathrm{mT}, 10 \mathrm{~Hz}$ magnetic field conditions. The results of this study confirmed that a magnetic field strength of $0.15 \mathrm{Gs}$, which has a promoting effect on bone healing, could prevent interruptions of the hormonal balance of high intensity.

Bone histomorphometry is a newly developed quantitative research method of bone tissue. It can transform two-dimensional images of bone tissue slices into quantity data as well as realize bone structure change from bone tissue and cellular levels. This study investigated the influence of PEMF on bone turnover in disuse osteoporosis from the bone histomorphometry angle. The experimental results show that, in the 4 weeks after the PEMF or ALN treatment, the \% Tb-Ar, Tb-N, and Tb-Sp parameters of 


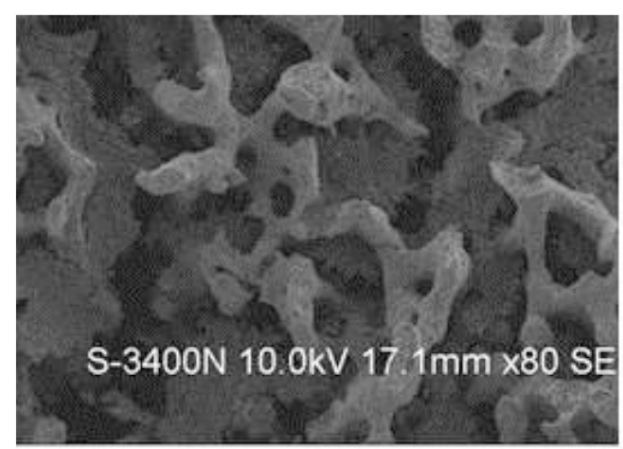

A. DOP group 2 week

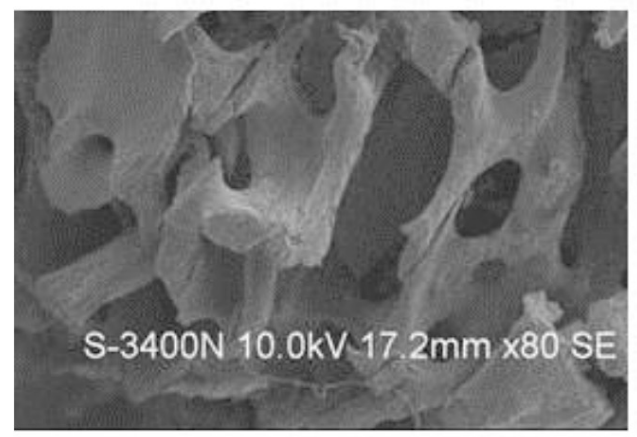

C.ALN group 4 week

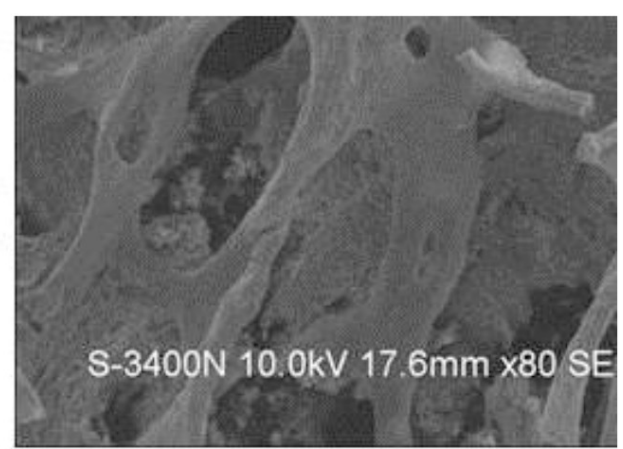

B.DOP group 4 week

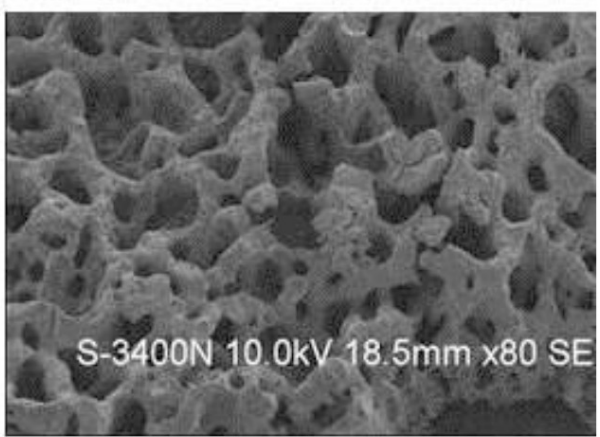

D. INT group 4 week

Fig. 3. The variation of the bone histomorphometry during the PEMF treatment.

the ALN group and PEMF group rats obviously changed. The bone microstructure changed, the area ratio and volume of the trabecular bone increased, and the average interval width of the trabecular bone decreased obviously. After a continuous 8 weeks of treatment, the Tb-Sp of the PEMF group was significantly lower, and the data indicated that, PEMF and ALN can improve disuse osteoporosis by changing the osseous tissue microstructure, quantity, structure, and the nature of bone trabecular, which achieves disuse osteoporosis treatment.

Throughout the treatment process, the Tb-Th of the ALN group and PEMF group showed no significant difference as compared with the DOP group. This may be due to the compensatory thickening of the trabecular bone in the DOP group, resulting in a similar trend with the treatment group.

BGP is a non-collagen that is specifically synthetized and secreted by osteoblasts and is a biochemical criterion that indirectly reflects bone metabolism [17]. BGP combines with hydroxylapatite and stabilizes its conformation in the presence of calcium ions; it is considered to be a marker of characteristic osteoblast differentiation [18].

In this research, the BGP concentrations of the rest groups were significantly increased as compared with that of the INT group after modeling. A significant increase in the bone biochemical markers indicated that osteoblast activity was enhanced in the disuse osteoporosis model. Disuse osteoporosis promoted bone absorption and bone formation simultaneously. The degree of bone absorption is much larger than that of bone formation, resulting in the formation of bone loss and osteoporosis. After modeling, the ALN group sustained reduced concentrations of BGP, and the effects of the ALN treatment were confirmed. Data suggested that alendronate may decrease osteoclast function, which inhibits bone 


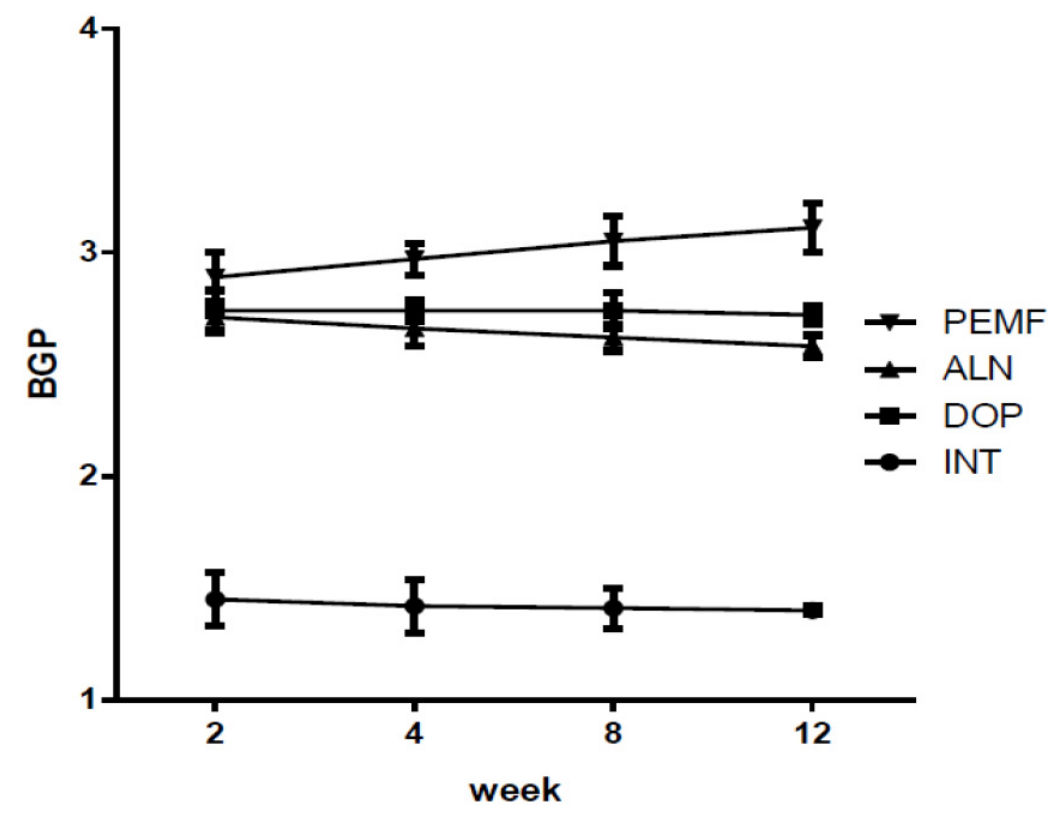

Fig. 4. Serum BGP concentrations of different rat groups at each time point after modeling parameters.

resorption. The serum BGP concentration of the PEMF group increased, and there was a significant difference as compared with the ALN group $(P<0.01)$. In fact, PEMF is better than drugs in enhancing osteoblast function. PEMF primarily prevented and treated disuse osteoporosis disease through promoting bone formation, and this view was confirmed by the experimental results.

However, bone structure mechanics and bone density should be studied and recorded in order to provide complete information of the real-time state of the bone. In our study, only a continuous monitoring of bone microstructure was carried out; thus, it does not fully reflect the state of the bone.

In summary, PEMF can prevent and treat DOP, and it can change the microstructure of bones. More specifically, BGP plays a crucial role in the process of improving DOP by PEMF. The experimental results indicate that for the prevention of DOP, drugs have a quick effect on DOP; however, in the long run, PEMF is less toxic and has fewer side effects, is safer, and is more reliable than drugs.

\section{Funding}

This study was funded by grants from the Science and Technology Talents Program of Harbin (2012RFXXS066, 2014RFXGJ041, 2014RFQGJ094, 2014RFXGJ035, the Department of Education Project of Heilongjiang province (11541147)) and The First Hospital of Harbin City talent introduction project (2013SYYRCYJ01-1).

\section{Conflict of interest}

None to report. 


\section{References}

[1] Shen WW, Zhao JH. Pulsed electromagnetic fields stimulation affects BMD and local factor production of rats with disuse osteoporosis [J]. Bioelectromagnetic. 2010; 31(2): 113-9.

[2] Zati A, Gnudi S, Mongiorgi R, Giardino R, Fini M, Valdrè G, Galliani I, Montagnani AM. Effects of pulsed magnetic fields in the therapy of osteoporosis induced by ovariectomy in the rat. Boll Soc Ital Biol Sper. 1993; Jul-Aug; 69(7-8): 469-75.

[3] Blain H, Chavassieux P, Portero-Muzy N, Bonnel F, Canovas F, Chammas M, Maury P, Delmas PD. Cortical and trabecular bone distribution in the femoral neck in osteoporosis and osteoarthritis [J]. Bone. 2008; 43(5): 862-8.

[4] Shen WW, Zhao JH. Pulsed electromagnetic fields stimulation affects BMD and local factor production of rats with disuse osteoporosis [J]. Bioelectromagnetic. 2010; 31(2): 113-9.

[5] Takano-Yamamoto T, Kawakami M, Sakuda M. Effect of a pulsing electromagnetic field on demineralized bone matrixinduced bone formation in a bony defect in the premaxilla of rats. J Dent Res. 1992; 71: 1920-1925.

[6] Bilotta TW, Zati A, Gnudi S, et al. Electromagnetic fields in the treatment of postmenopausal osteoporosis: An experimental study conducted by densitometric, dryash weight and metabolic analysis of bone tissue. Chir Organi Mou. 1994; 79: 309-313.

[7] Jee WS, Wronski TJ, Morey ER, Kimmels DB. Effects of spaceflight on trabecular bone in rats. Am J Phys. 1983; 244: R310-R316.

[8] Halloran BP, Bikle DD, Cone CM, Morey-Holton E. Glucocorticoids and inhibition of bone formation induced by skeletal unloading. Am J Phys. 1988; 244: E875-E879.

[9] Chang K, Chang WHS, Huang S, et al. Pulsed electromagnetic fields stimulation affects osteoclast formation by modulation of osteoprotegerin, RANK ligand and macrophage colony-stimulating factor. J Orthop Res. 2005; 23: 1308-14.

[10] Kasturi GC, Cifu DX, Adler RA. A review of osteoporosis: Part I. Impact, pathophysiology, diagnosis and unique role of the physiatrist. PM R. 2009; 1: 254-260.

[11] Smith EM, Comiskey CM, Carroll AM. A study of bone mineral density in adults with disability. Arch Phys Med Rehabil. 2009; 90: 1127-1135.

[12] Shinchuk LM, Morse L, Huancahuari N, Arum S, Chen TC, Holick MF. Vitamin D deficiency and osteoporosis in rehabilitation in-patients. Arch Phys Med Rehabil. 2006; 87: 904-908.

[13] Gonzalez-Riola J, Pamies JA, Hernandez ER, Revilla M, Seco C, Villa LF, et al. Influence of electromagnetic fields on bone mass and growth in developing rats: a morphometric, densitometric and histomorphometric study. Calcif Tissue Int. 1997; 60: 533-7.

[14] Yamaguchi DT, Huang J, Ma D, Wang PKC. Inhibition of gap junctionintercellular communication by extremely lowfrequency electromagnetic fields in osteoblast-like models is dependent on cell differentiation. J Cell Physiol. 2002; 190: $180-8$.

[15] Oxlund SB, Ortoft G, Andreassen TT, Oxlund H. Low-intensity, high-frequency vibration appears to prevent the decrease in strength of the femur and tibia associated with ovariectomy of adults rats. Bone. 2003; 32: 69-77.

[16] Judex S, Lei X, Han D, Rubin C. Low-magnitude mechanical signals that stimulate bone formation in the ovariectomized rat are dependent on the applied frequency but not on the strain magnitude. J Biomech. 2007; 6: 1333-9.

[17] Zhu X, Luo J, Chen X, Wang J, Wang G, Li H, Xu Y, Feng J, Tu H. Expression characteristic and significance of interleukin-6, nuclear factor kappa beta, and bone formation markers in rat models of osteoporosis [J]. Transl Res. 2008; 152(1): 18-23.

[18] Levy MM, Joyner CJ, Virdi AS. Osteoprogenitor cells of mature human skeletal muscle tissue: an in vitro study [J]. Bone. 2001; 29(4): 317-322. 